\title{
Preservación de Placenta Humana. Técnica Anatómica
}

\author{
Preservation of Human Placenta. Anatomical Technique
}

\author{
"María Fabiola Bustamante A.; "Ruth Haydée Prieto Gómez \& ** Octavio Binvignat Gutiérrez
}

\begin{abstract}
BUSTAMANTE, A. M. F.; PRIETO, G. R. H. \& BINVIGNAT, G. O. Preservación de placenta humana. Técnica anatómica. Int. J. Morphol., 25(3):545-548, 2007.
\end{abstract}

RESUMEN: Las técnicas de fijación, conservación y preservación, con fines didácticos y académicos, buscan la manera de encontrar sustancias que mantengan, dentro de lo posible, el estado natural del cuerpo u órganos, penetrando en los tejidos y permitiendo un fácil montaje, proporcionando información detallada y una visión tridimensional de las estructuras.

Se planteó como objetivo del trabajo la visualización de la angioarquitectura de placenta humana, de gestación a término, partos normales, únicos, perteneciente al Servicio de Ginecología y Obstetricia, Unidad de Partos, del Hospital Hernán Enríquez Aravena, de Temuco, IX Región, Chile. Estas fueron inyectadas a través de los vasos placentarios con resina acrílica autopolimerizable, realizando luego, una corrosión por 11 días con HCL al 32,4\%, siendo hidrolizado completamente el parénquima y el estroma de la placenta, obteniéndose el molde de los vasos.

Nuestros resultados confirmaron que esta técnica es útil en docencia e investigación, dado los bajos costos y excelente visualización macroscópica de la trama vascular de la placenta humana.

PALABRAS CLAVE: Angioarquitectura; Corrosión; Placenta humana.

\section{INTRODUCCIÓN}

La preocupación por la conservación de los cuerpos humanos y piezas anatómicas, ha sido constante desde el principio de la humanidad, ya a partir del siglo XVI surge con fines didácticos y académicos. La fijación, conservación y preservación son los pilares fundamentales para que una pieza anatómica quede semejante al órgano fresco. Muchas han sido las técnicas y fórmulas químicas empleadas a lo largo del tiempo, que si bien consiguieron algunos resultados parciales, no resolvieron satisfactoriamente el problema de la conservación (Correa, 2001). La fijación es un método que inmoviliza las estructuras celulares de los órganos impidiendo la autólisis y que permite las observaciones posteriores (Coliez, 1927).

El objetivo de este artículo es presentar un método económico, de relevancia para la docencia e investigación como es la repleción-corrosión, usando resinas acrílicas autopolimerizables, que permiten elaborar moldes de los vasos sanguíneos y visualizar la angioarquitectura de la placenta humana.
Las técnicas de conservación experimentaron un importante avance al emplearse diversas sustancias químicas debido principalmente a investigadores como Hunter (17181783), quien utilizó el alcohol como medio de fijación y conservación; Dionis empleó el ácido tánico para evitar el crecimiento de hongos, Chaussier (1746-1828), útilizó el sublimado o bicloruro de mercurio para evitar la putrefacción y favorecer la modificación; Ritter (1714-1784) utilizó arsénico, Scheele (1742-1786) aplicó la glicerina para la conservación de cadáveres, von Hofmann (1818-1892) químico alemán descubrió el formol el año 1868. (Hildebrand, 1989). Con el uso del formol se produce una innovación en las técnicas de fijación de tejidos, tanto que a la fecha ha sido base de la conservación y fijación de piezas anatómicas, como en la preparación de piezas para estudios de histología. Estas propiedades de fijación y conservación, unidas a una rápida y fácil difusión tisular, hacen que, en la actualidad, se esté empleando como base de las innumerables fórmulas que existen para estos fines (Hildebrand). Sin embargo, el uso del formol ha planteado graves problemas referentes tanto a la seguri-

* Facultad de Medicina, Universidad de La Frontera, Temuco, Chile.

${ }^{* *}$ Faculdade da Serra Gaúcha, (RS), Brasil. 
dad de los usuarios y manipuladores como a los precarios resultados obtenidos en la calidad de los tejidos conservados. Antiguamente, se empleaban recipientes de cristal transparente llenos de líquido conservador, donde se sumergía la pieza anatómica. De acuerdo a su densidad ésta podía flotar en el líquido o caer al fondo del recipiente. La mayoría de las veces, la pieza anatómica se alzaba con unas cuerdas o se ponía sobre una placa de cristal transparente coloreada en azul oscuro o en negro si se quería obtener contraste. Actualmente se prefiere presentar las muestras en recipientes de material plástico transparente que son mucho más ligeros y menos frágiles (Kessemberg, 1928; Fenton, 1956 y Audette, 1965).

El uso de material plástico facilita la conservación de la forma exacta de objetos pequeños y simplifica su manipulación posterior. En general esta forma de conservación es similar a las técnicas de inclusión y de recubrimiento o envoltura que son los casos particulares de conservación que permiten inmovilizar todas las estructuras de forma definitiva. La protección, de los organismos muertos, contra el medio ambiente, la humedad de los agentes que favorecen la descomposición, es realizada mediante una capa de celuloide o sea una película impermeable. El método de inclusión mencionado, necesita usar materias plásticas transparentes, duras, de retracción leve al secado. Las resinas sintéticas de poliéster poseen esas propiedades al igual que ciertas resinas metacrílicas, aunque más teñidas. (Bassett, 1947).

\section{MATERIAL Y MÉTODO}

Utilizamos las técnicas anatómicas de repleción y corrosión en placenta humana para observar los vasos de este órgano, inyectando resina acrílica, y corrosión con ácido clorhídrico, para visualizar su angioarquitectura.

Se usaron ocho placentas humanas, de partos normales, de gestación a término, únicos, promedio de peso de la placenta superior a $500 \mathrm{~g}$, obtenidas en un tiempo menor a 5 horas post-alumbramiento. Conservadas en solución acuosa y refrigeradas a $4^{\circ} \mathrm{C}$, obtenidas del Hospital Hernán Henríquez Aravena, Temuco, IX Región, Chile.

Para la realización de la técnica se utilizaron los siguientes instrumentales, materiales e insumos: Sondas nasogástricas siliconadas $\mathrm{N}^{\circ} 12-16$, seda quirúrgica, material de disección: (Pinzas anatómicas, bisturí, portabisturí, tijeras, pinzas hemostáticas), guantes de procedimiento, mascarillas de alta eficiencia, refrigerador doméstico, contenedores plásticos, Unidades refrigerantes, jeringas desechables de 50-60cc, acrílico autopolimerizable (Productos Dentales Marche $\left.{ }^{\circledR}\right)$ pintura acrílica roja y azul como colorante de la resina, guantes de goma, mascarilla con filtro para gases, campana de extracción y ácido clorhídrico (HCL) al 32,4\%.

Técnica de repleción-corrosión química $\mathrm{HCl}$ al 32,4 \%:

1. Obtención de la muestra.

2. Canulación y fijación de cánulas.

3. Lavado con el monómero de la resina, hasta limpieza total de vasos.

4. Preparación de acrílico y repleción manual.

5. Refrigeración a $4^{\circ} \mathrm{C}$, durante 2 días.

6. Corrosión con $\mathrm{HCl}$ al 32,4\%, durante 7 días.

7. Cambio de baño de $\mathrm{HCl}$ al 32,4\%, durante 4 días, siendo hidrolizado completamente el parénquima y el estroma de la placenta, obteniéndose el molde de las vías inyectadas.

8. Lavado continuo por 4 horas.

9. Aireación 7 días y montaje en caja de vidrio.

\section{RESULTADOS}

Los resultados de la técnica de repleción-corrosión son los siguientes:

En la cara fetal (placa corial en estado fresco) de la placenta humana se evidencia la anastomosis vecina al cordón umbilical. Las ramas de estas arterias se dirigen individualmente a los cotiledones, sin entrelazarse, las que constituyen los ejes de los troncos vellositarios, hecho de sumo interés en el estudio de la circulación placentaria (Fig. 1).

Por otra parte, en una perspectiva de la cara materna (placa basal en estado fresco) de la placenta humana se puede observar el grado de inyección de los vasos sanguíneos con acrílico dental, que fue previamente corroída, apreciándose el excelente grado de penetración de esta sustancia en el lumen de los pequeños vasos de la trama vascular. Se observa también la vena umbilical (en azul) dando rama a cada uno de los cotiledones.

Es interesante ver a través del montaje en acrílico, la unión del cordón umbilical con la placenta, evidenciando las arterias y la vena. Llama la atención la anastomosis que existe en este punto entre las arterias, las que como se ve dan origen a las arterias de los troncos vellositarios en cada uno de los cotiledones, los que a su vez se capilarizan finalmente en las vellosidades coriónicas libres (visión microscópica). La ausencia de los tabiques o septos intercotiledonarios (presentes en fresco) hace lucir la cara materna como una sola unidad, sin embargo, la cuidadosa observación permite distinguir claramente pequeños vasos agrupados en unidades. 


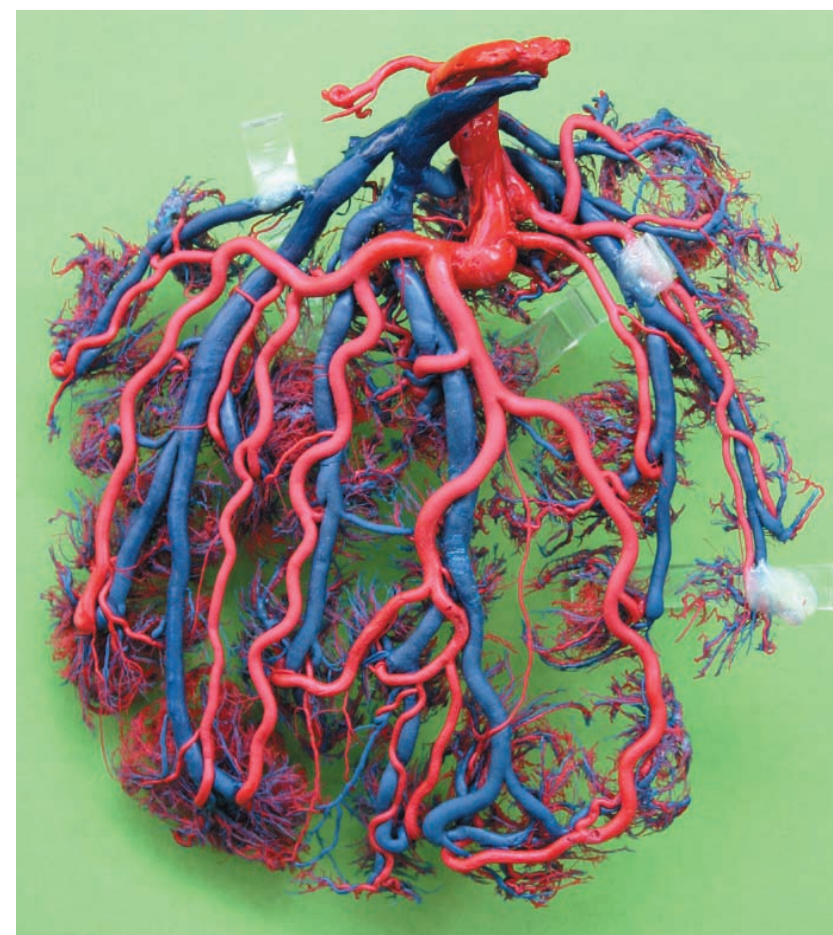

Fig. 1. Placenta humana. Técnica de repleción-corrosión.

\section{DISCUSIÓN}

Al realizar la repleción de los vasos sanguíneos de la placenta humana se comparó con la técnica de disección, una ventaja encontrada es que reduce considerablemente la pro- babilidad de seccionar por error una parte de la muestra, a diferencia de la disección. (Sandi, 1958). Además, la técnica de repleción, permite llegar en un solo paso a todos los planos de la muestra, destacando la posición de los ejes de los troncos vellositarios, hecho de sumo interés en el estudio de la circulación placentaria. (Carlson, 2000). Otra ventaja es que la luz no altera la conservación de la muestra, solo requiere de un montaje apropiado para su adecuada manipulación. También la visualización macroscópica de la trama vascular de la placenta se hace más comprensible para los estudiantes. (Obrien, \& Gunnar, 1995). Importante ventaja en la ejecución de la técnica, son el costo asequible y resultados rápidos de obtener. (Sarmento et al., 1995).

El cuidado que se debe tener con la resina, debido a su rápida polimerización, (Obrien, \& Gunnar, 1995), es que restringe el tiempo de inyección a unos cuantos segundos. Además, no olvidar que los procedimientos de la técnica en el laboratorio requieren del uso de medidas de bioseguridad según normas. (Tompsett, 1959).

Coincidimos con Matamala et al., (1984), que esta técnica es excelente para trabajar en docencia e investigación, además la resina acrílica es de bajo costo y fácil de adquirir para cualquier Centro de Investigación y docencia.

El objetivo del trabajo permitió la observación macroscópica y tridimensional de la red vascular de la placenta humana. Este tipo de modelos es una excelente herramienta metodológica como parte del proceso de aprendizaje para alumnos de pre y postgrado.

BUSTAMANTE, A. M. F.; PRIETO, G. R. H. \& BINVIGNAT, G. O. Preservation of the human placenta. Anatomical technique. Int. J. Morphol., 25(3):545-548, 2007.

SUMMARY: The fixation, conservation and preservation techniques, with academic and didactic aims, look for the way to find substances that maintain, as far as it is possible, the natural state of the body or organs, permeating the tissues, allowing an easy assembly, providing a detailed information and offering a three-dimensional view of the structures. The objective of the research was the view of angioarchitecture of the human placenta. The samples used were taken from the Servicio de Ginecología y Obstetricia, Unidad de Partos, del Hospital Hernán Enríquez Aravena, de Temuco, IX Región, Chile. All placentas were of a completed gestation condition, of single and normal childbirths. The placentas were injected with hardening solutions, and subject to corrosion with hydrochloric acid $32,4 \%$. This allowed the creation of a pattern of arteries and veins. Our results confirm that this technique can be useful in teaching and investigation, in view of the low costs and excellent results in the macroscopic visualization of the vascular plot of the human placenta.

KEY WORDS: Angioarchitecture; Corrosion; Human placenta.

\section{REFERENCIAS BIBLIOGRÁFICAS}

Audette, L. G. A method of construction plastic display. Anat. Rec., 163:383-7, 1965.

Bassett, D. Ethyl methacrylate as a preserving medium for gross anatomical serial sections. Anat. Rec., 99(2):145-50, 1947.
Carlson, B. Embriología humana y biología del desarrollo. $2^{\mathrm{a} \text { ed }}$. Harcourt, Madrid, 2000.

Coliez, A. De la conservation artificialle des cadavres. Historique Technique moderne des emvaumements. Th. Paris, 1927. 
Correa, A. Profesor Titular Cátedra de Anatomía Unidad de medicina veterinaria. Universidad de Granma, 2001, www.portalveterinaria.com.

Fenton, E. A design for perfex suseus, J. Med. Lab. Technol., 15:249-232, 1956.

Hildebrand, M. Anatomical preparations. University of California Press. Beakeley and Los Angeles, 1968.

Kessemberg, J. M. Containers for sagital and frontal sections of cadavers. Anat. Nac., 42:376-9, 1928.

Matamala, F.; López, P.; Iturriaga, P. \& Chávez, R. Contribución al estudio de la resina poliéster para su uso en docencia e investigación anatómica. An. Anat. Normal, 2:138-42, 1984.

Obrien, W. \& Gunnar, R. Un método rápido para muestras inyectadas con resinas poliéster. Rev. Chil. Anat., 13(1):11-6, 1995.
Sandi, T. Modelo de cuba para conservação de cadáveres. Folia Clin. Path., 2:92-5, 1958.

Tompsett, D. Improvements in corrosion casting techniques. An. R. Coll. Surg. Engl., 24(2):110-23, 1959.

Sarmento, A.; Olave, E.; Gabrielli, C. \& Pardi, P. La belleza de la diafanización: Un método rápido para muestras inyectadas con resina poliéster. Rev. Chil. Anat., 13(1):116, 1995.

Dirección para correspondencia:

Prof. María Fabiola Bustamante A.

Facultad de Medicina

Universidad de La Frontera

Temuco - CHILE

Recibido : 16-09-2007

Aceptado: 23-05-2007 